\title{
T2K-WAGASCl: First physics run of the WAGASCI-BabyMIND detector with full setup
}

\author{
Giorgio Pintaudi* ${ }^{*}$ \\ Yokohama National University, Japan \\ E-mail: giorgio-pintaudi-kx@ynu.jp
}

The WAGASCI project aims to perform a study of neutrino-nucleus interactions at the J-PARC accelerator in Japan with a new fine-grained neutrino detector (WAGASCI module) coupled with muon range detectors (WallMRD and BabyMIND).

The WAGASCI module main target for neutrinos is purified water. A hollow cuboid lattice made of scintillators bars is used to detect charged particles coming out of the neutrino interaction points. Measurement in wide phase space becomes possible by the combination of the WAGASCI modules, side and downstream muon range detectors. The downstream-MRD, the so-called Baby MIND detector, also works as a magnet and provides charge identification capability as well as magnetic momentum measurement for high energy muons.

The detectors have been constructed and have been commissioned in the J-PARC T59 experiment and the CERN neutrino platform. They are going to be exposed to the same neutrino beam as the T2K experiment at an off-axis angle of 1.5 degrees. The collaboration will be ready to collect physics data by November 2019. The first milestone for the WAGASCI project is to have at least $0.5 \times 10^{20}$ POT (protons on target) in neutrino mode and the same amount in anti-neutrino mode (figure 7).

We will provide inclusive and exclusive differential cross sections of the charged current neutrino and antineutrino interactions with water and hydrocarbon with slightly higher neutrino energy and with a wider angle acceptance than T2K-ND280.

By combining our measurements with those from ND280, model-independent extraction of the cross-section for narrow energy spread becomes possible. These measurements would improve the understanding of the neutrino-nucleus interaction at around $1 \mathrm{GeV}$ and thus contributing to reducing one of the most significant uncertainties sources of the T2K experiment.

XXIX International Symposium on Lepton Photon Interactions at High Energies - LeptonPhoton2019 August 5-10, 2019

Toronto, Canada

\footnotetext{
* Speaker.

${ }^{\dagger}$ on behalf of the WAGASCI collaboration
} 


\section{The WAGASCI experiment}

WAter Grid And SCIntillator (WAGASCI[1]) is a new neutrino cross-section experiment located in Japan at the J-PARC facility. It is exposed to the very same neutrino beam as the T2K experiment but at a different off-axis angle of $1.5 \mathrm{deg}$. It is located in the same building as the off-axis T2K Near Detector (ND280) and at the same distance of 280m from the beam target.

Its short-term aim is to measure with high precision the neutrino- $\mathrm{H} 2 \mathrm{O}$ and neutrino- $\mathrm{CH}$ crosssections and their ratio. Its strong points are:

- the neutrino passive target (WAGASCI detector) is mostly water (Water:CH 80:20), same as the T2K far detector SuperKamiokande;

- it has good sensitivity to side-going muons thanks to the two Side Muon Range Detectors (WallMRD);

- it has a fine-grained grid-like structure that gives a good 3D track reconstruction efficiency (figure 1);

- Use the Baby Magnetised Iron Neutrino Detector (BabyMIND[2]) to discriminate the charge of the forward going muons.

Among its longer-term aims are:

- use this cross-section measurement to decrease the T2K systematic error;

- improve the understanding of the neutrino-nucleon cross-section and final state interaction models;

- the substantial difference in the neutrino beam peak energy with respect to ND280 enables to develop and test new analysis techniques to narrow down the neutrino initial energy by comparing the results of the two experiments (figure 2);

- improve, test and benchmark the SPIROC chip (WAGASCI)and FEB board (BabyMIND) and the surrounding electronics;

- develop and test a new data acquisition software framework (based on the MIDAS and Pyrame programs);

The light from each scintillator bar is collected by a wave-length shifting fiber and read out by a Hamamatsu MPPC (S13660 for the WAGASCI module). The low dark noise and crosstalk rates of this MPPC model enable to take data with a low-ish threshold of 1.5 or 2.5 photo equivalent units and continuously calibrate the detector using the dark noise hits between the beam spills.

\section{Commisioning run}

The commissioning run (spring 2018), on top of testing the equipment, led to a measurements of the flux integrated differential and total $\mathrm{CC} 0 \pi 0 \mathrm{p}$ cross sections on $\mathrm{H} 2 \mathrm{O}$ and $\mathrm{CH}$ and their ratio, using one WAGASCI module as the water target, a full scintillator module called Proton Module as the hydrocarbon target and an INGRID module as muon range detector (figures 3 and 4). 


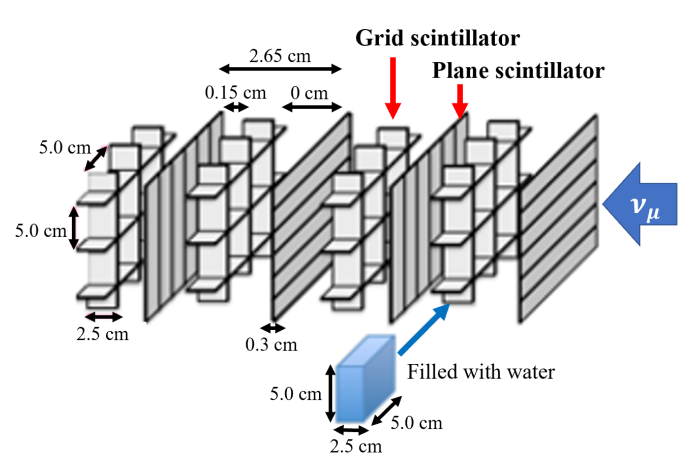

Figure 1: WAGASCI module inner structure (water $\sim 80 \%$, hydrocarbon $\sim 20 \%$ )

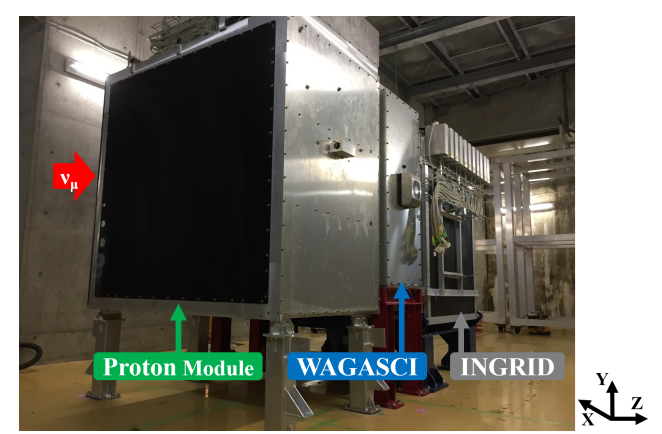

Figure 3: Front view of the commissioning setup.

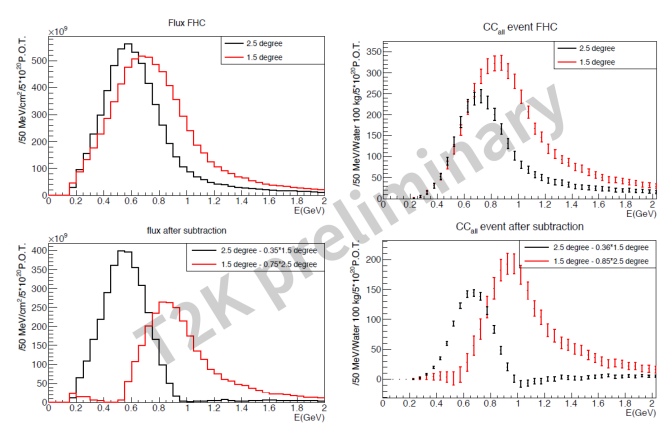

Figure 2: WAGASCI and ND280 neutrino flux and $\mathrm{CC}$ events distribution and their subtraction.

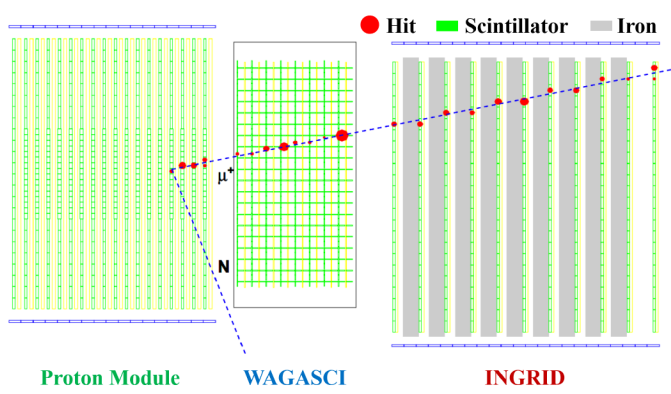

Figure 4: CCQE-like event display for the commissiong setup.

At the location for those detectors, the mean energy of the $\bar{v}_{\mu}$ component in the anti-neutrino beam is $860 \mathrm{MeV}$. The analysis used $7.91 \times 10^{20}$ POT of anti-neutrino mode data taken in T2K RUN9. The CC0 $\pi 0$ p signal was defined as follows: one muon + no charged pion + no proton.

Due to the detector acceptance of WAGASCI and the Proton Module, the following restrictions to the final state particles were applied:

- protons: $p_{p}>600 \mathrm{MeV} / \mathrm{c}$ and $\theta_{p}<70$ degrees

- pions: $p_{\pi}>200 \mathrm{MeV} / \mathrm{c}$ and $\theta_{\pi}<70$ degrees

- muons: $p_{\mu}>400 \mathrm{MeV} / \mathrm{c}$ and $\theta_{\mu}<30$ degrees

where $p_{x}$ is the momentum and $\theta_{x}$ is the angle with respect to the beam direction for the particle $x$.

Since no magnetic field was applied to those detectors, the $v_{\mu}$ interactions are the dominant background events for the $\bar{v}_{\mu}$ cross-section measurement. By including $v_{\mu} \mathrm{CC} 0 \pi 0 \mathrm{p}$ events into the signal, the signal purity is increased and a better experimental precision is achieved (figure 5).

\section{First Physics run with full setup}

Aiming at the next physics run starting from November 2019 to January 2020, we installed many new detectors as shown in figure 6. There are two WAGASCI modules, one PROTON mod- 

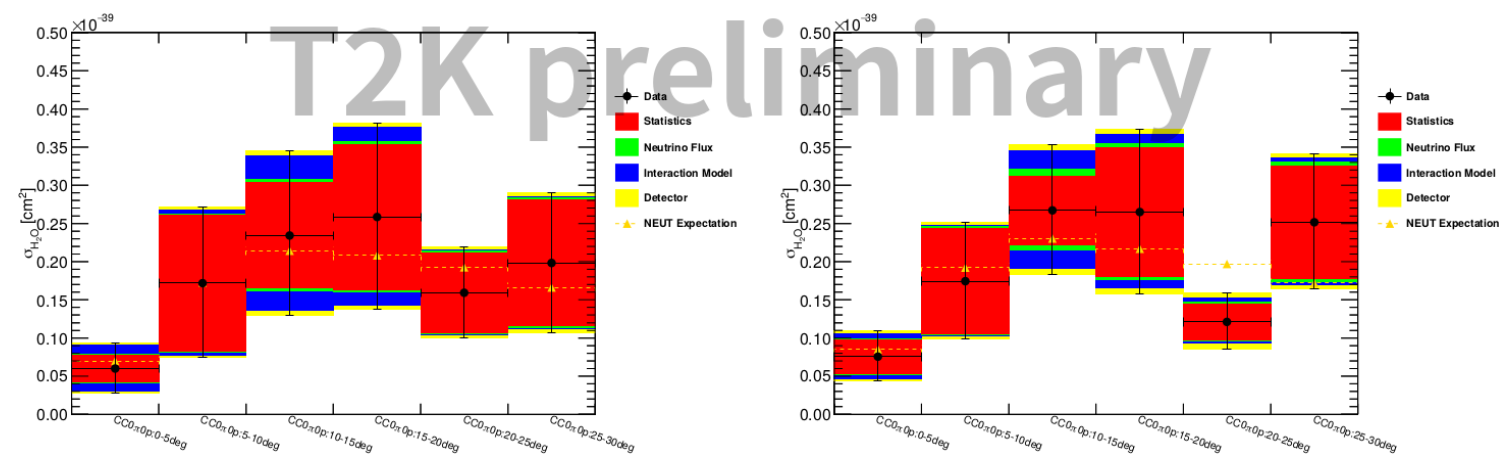

Figure 5: The differential CCO $\pi 0 \mathrm{p} \bar{v}_{\mu}$ (left) and $\bar{v}_{\mu}+v_{\mu}$ (right) cross sections on $\mathrm{H} 2 \mathrm{O}$ with respect to the muon scattering angle satisfying the restrictions above.

ule, two WallSMRD, and the BabyMIND detector. The INGRID module is replaced by the much more capable BabyMIND detector.

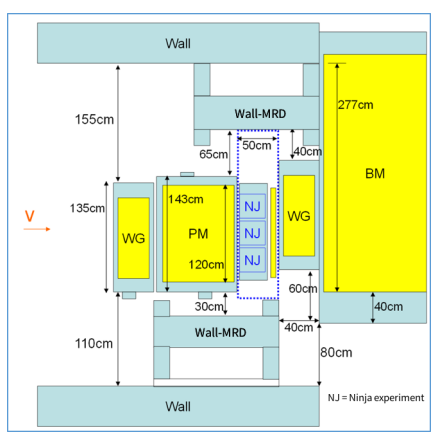

Figure 6: Location of the detectors in the final setup. The "NJ" label refers to the NINJA experiment (not part of WAGASCI).

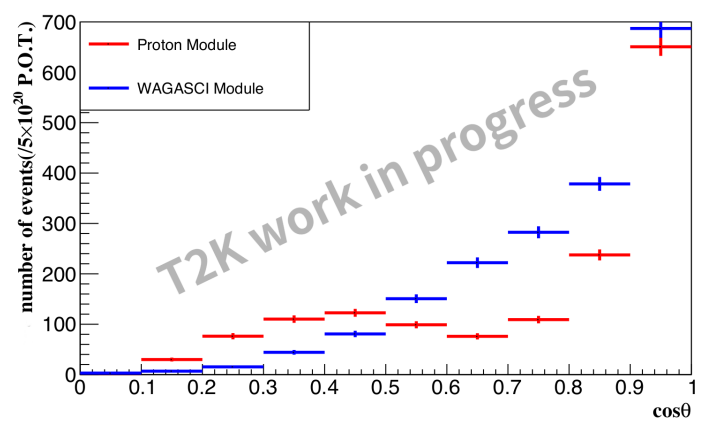

Figure 7: Number of expected events for $5 \times 10^{20} \mathrm{POT}$ in anti-neutrino mode with respect to the muon scattering angle. The fiducial volume and other cuts used here are pretty conservative.

Another improvement with respect to the previous runs is the brand new DAQ software, based on the Pyrame and MIDAS frameworks. The DAQ software was developed with stability and easeof-use in mind.

Because the BabyMIND electronics will be the base on top of which the new ND280 upgrade electronics is going to be developed, and the WAGASCI and ND280 DAQ software bear many similarities, the knowledge acquired while building and operating our detectors would prove useful for the ND280 upgrade endeavor as well.

\section{References}

[1] T. K. et al., Water/CH Neutrino Cross Section Measurement at J-PARC (WAGASCI Experiment), ch. 3. JPS Conf. Proc., 2015. 10.7566/JPSCP.8.023003.

[2] M. Antonova et al., Baby MIND: A Magnetised Spectrometer for the WAGASCI Experiment, in Proceedings, Prospects in Neutrino Physics (NuPhys2016): London, UK, December 12-14, 2016, 2017, 1704.08079 , http://lss.fnal.gov/archive/2017/conf/fermilab-conf-17-270-apc.pdf. 